\title{
Counting Fixed-Height Tatami Tilings
}

\author{
Frank Ruskey* \\ Department of Computer Science \\ University of Victoria \\ PO BOX 3055, Victoria BC, Canada V8W 3P6 \\ ruskey@cs.uvic.ca \\ Jennifer Woodcock ${ }^{\dagger}$ \\ Department of Computer Science \\ University of Victoria \\ PO BOX 3055, Victoria BC, Canada V8W 3P6 \\ jwoodcoc@cs.uvic.ca \\ Submitted: Jul 10, 2009; Accepted: Sep 30, 2009; Published: Oct 12, 2009 \\ Mathematics Subject Classification: 05B45, 05A19
}

\begin{abstract}
A tatami tiling is an arrangement of $1 \times 2$ dominoes (or mats) in a rectangle with $m$ rows and $n$ columns, subject to the constraint that no four corners meet at a point. For fixed $m$ we present and expand upon Dean Hickerson's combinatorial decomposition of the set of tatami tilings - a decomposition that allows them to be viewed as certain classes of restricted compositions when $n \geqslant m$. Using this decomposition we find the ordinary generating functions of both unrestricted and inequivalent tatami tilings that fit in a rectangle with $m$ rows and $n$ columns, for fixed $m$ and $n \geqslant m$. This allows us to verify a modified version of a conjecture of Knuth. Finally, we give explicit solutions for the count of tatami tilings, in the form of sums of binomial coefficients.
\end{abstract}

\section{Introduction}

In the dimer problem one wishes to count the number of different dimer configurations ways to cover an $m$ by $n$ rectangle by 1 by 2 tiles. An equivalent problem is counting the number of perfect matchings in a grid graph. Many papers have been written about the

\footnotetext{
${ }^{*}$ Research supported in part by Natural Sciences and Engineering Research Council of Canada (NSERC) Discovery and Equipment grants.

${ }^{\dagger}$ Research supported by an NSERC PGS-D scholarship.
} 

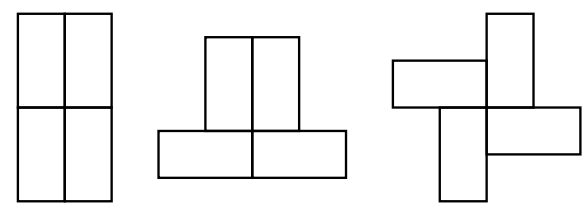

Figure 1: Some forbidden configurations for tatami tilings
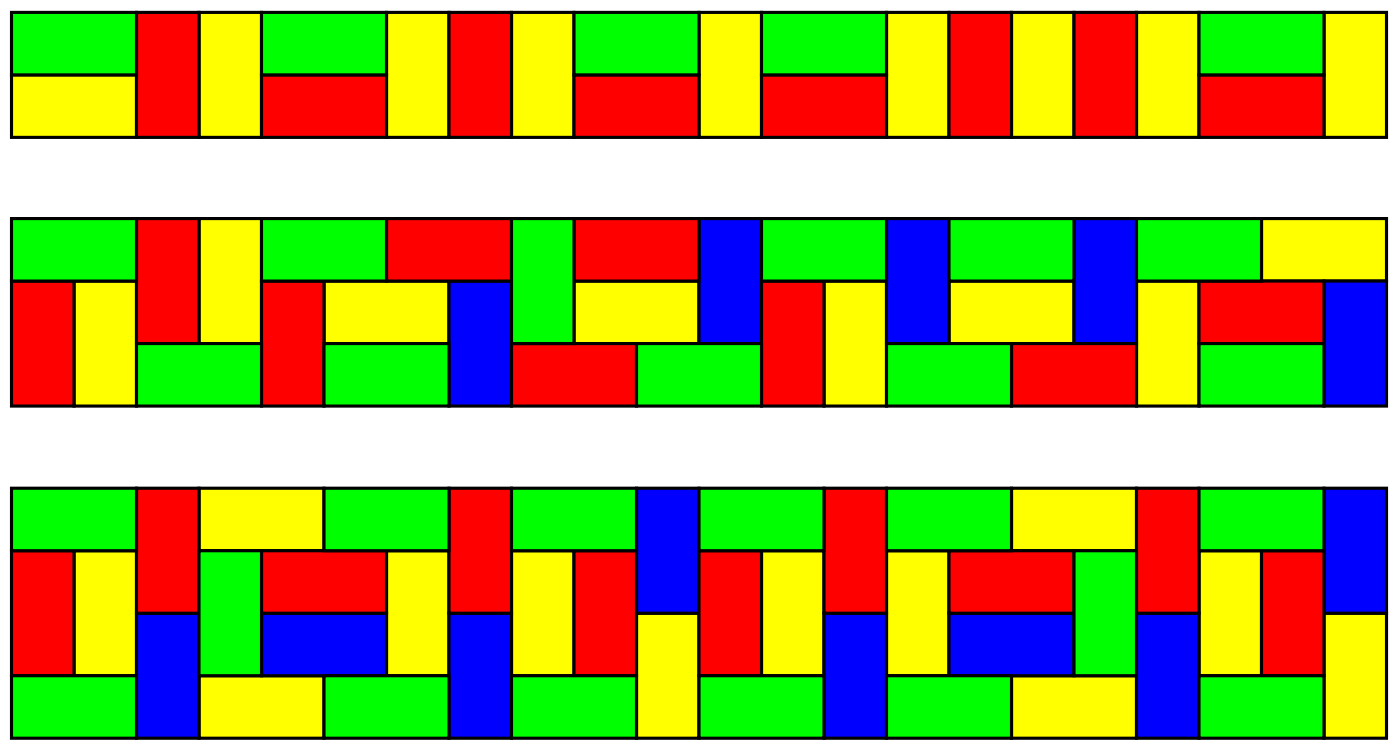

Figure 2: Typical tatami tilings for $m=2,3,4$.

dimer problem and its variants. See, for example Read [5] or Section 10.1, "The Dimer Problem and Perfect Matchings," of Aigner [1]. Dimer configurations are also sometimes called domino tilings and we will use the word "tiling" in this paper. All of our tilings will be of the type defined below.

Definition 1.1. A tatami tiling is a domino tiling which satisfies the tatami property that no four tiles meet at a point.

A japanese tatami mat usually has a width to length ratio of $1: 2$, just like 1 by 2 tiles. These mats are, traditionally, used to tile a rectangular floor using a tatami tiling. Figure 1 shows some forbidden configurations for tatami tilings and Figure 2 shows examples of tatami tilings for $m=2,3,4$.

In this paper we are concerned with tatami tilings where the height $m$ is fixed. Figure 2 shows typical tatami tilings with $m=2,3,4$. Let $T(m, n)$ denote the number of height $m$, width $n$ tatami tilings. In the solution to problem 7.1.4.215 of Knuth [4], he conjectures that the ordinary generating function for the number of tatami tilings of an $m$ by $n$ 
rectangle with $m$ even and $0 \leqslant m-2 \leqslant n$ is

$$
K(z)=\frac{(1+z)^{2}\left(z^{m-2}+z^{m}\right)}{1-z^{m-1}-z^{m+1}}
$$

By his conjecture, the coefficient of $z^{n}$ in $K(z)$ equal to $T(m, n)$ whenever $m$ is even, $m \geqslant 2$, and $n \geqslant m-2$.

Hickerson [3], provides a construction for tatami tilings of $m$ by $n$ rectangles for all values of $m$, which we will use to show that Knuth's generating function is mainly correct. However, it only generates the number of tatami tilings of an $m$ by $n$ rectangle whenever $m$ is even and $4 \leqslant m \leqslant n$ or $6 \leqslant m-2 \leqslant n$, and not when $m=2$ or when $n=4$ and $m=2$ or $m=3$, or when $n=6$ and $m=4$, as was conjectured.

In Section 2 we describe Hickerson's structural characterization of $m$ by $n$ tatami tilings for $1 \leqslant m \leqslant n[3]$. Section 3 explains how to construct generating functions for $m$ by $n$ tilings with $m=2$ (Section 3.1), $m$ odd and $3 \leqslant m \leqslant n$ (Section 3.2), and $m$ even and $2 \leqslant m \leqslant n$ (Section 3.3). Section 3.4 discusses the range of validity of $K(z)$.

The aforementioned sections discuss tatami tilings without consideration of equivalence by reflection or rotation. Thus in Section 4 we construct generating functions for inequivalent tatami timings. As in Section 3, we construct separate generating functions for $m=2$ (Section 4.1), for $m$ odd and $3 \leqslant m \leqslant n$ (Section 4.2), and for $m$ even and $4 \leqslant m \leqslant n$ (Section 4.3).

Finally, in section 5 we provide tables generated by the generating functions presented here as well as a summary of the results.

\section{Structure of Tilings}

Theorem 2.1. The "triangular" corners of a rectangle cannot be tiled.

Proof. This is illustrated without loss of generality by Figure 3.

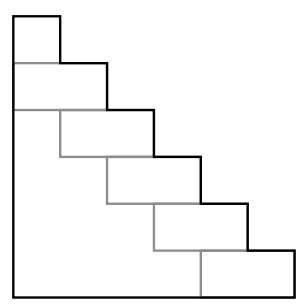

Figure 3: The lower left triangle of a rectangle which is impossible to tile.

Theorem 2.2. A tatami tiling of an $m$ by $n$ rectangle with $1 \leqslant m \leqslant n$ has no horizontal tile touching the left edge of the rectangle that is not also touching either the top or bottom edge of the rectangle. 
Proof. The cases where $m=1$ and $m=2$ are trivial since a horizontal tile that touches the left edge of the rectangle must also touch the top or bottom. Consider a tatami tiling of an $m$ by $n$ rectangle with $3 \leqslant m \leqslant n$ and suppose there was such a tile $t$ touching the left edge but not the top or bottom edge. There are two cases: either the row containing $t$ is completely filled with horizontal tiles or it isn't.

case 1 Assume without loss of generality that row $r$ with $\left\lfloor\frac{m}{2}\right\rfloor<r<m$ contains only horizontal tiles. Note that this means $n$ must be even. Thus $r$ is in the bottom half of the rectangle and $r$ contains exactly $\frac{n}{2}$ tiles. Because of the tatami property, for $1 \leqslant i \leqslant m-r$, row $r+i$ must contain $\frac{n}{2}-i$ contiguous horizontal tiles starting in column $i+1$. Since $n \geqslant m$ and $r>\left\lfloor\frac{m}{2}\right\rfloor$, row $m$ contains $\frac{n}{2}-m+r \geqslant\left\lceil\frac{m}{2}\right\rceil-m+r=r-\left\lfloor\frac{m}{2}\right\rfloor>0$ tiles. The remaining untiled portion of the bottom half of the rectangle contains two corners which cannot be tiled as shown in Theorem 2.1. Figure 4 illustrates these row tilings with row $r$ shown in black and rows $r+i$ for $1 \leqslant i \leqslant m-r$ shown in grey.

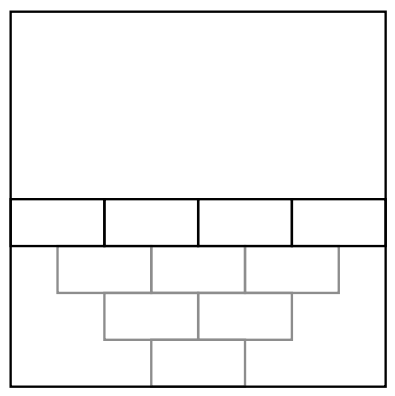

Figure 4: Forced tiling with a horizontal tile row.

case 2 Assume that row $r$ with $1<r<m$ contains at least one square that is covered by a vertical tile. Consider the leftmost vertical tile, $t$, in row $r$ and assume without loss of generality that $t$ covers row $r$ and row $r+1$ as shown in Figure 5 .

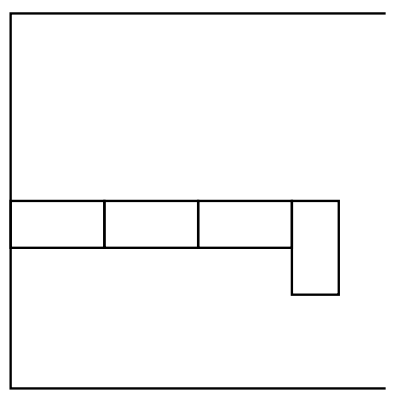

Figure 5: Leftmost vertical tile in a row with a horizontal tile on the left edge.

Now, as shown by the grey solid tiles in Figure 6, the two tiles above and to the right of $t$, and above and to the right of those two tiles, and so on, are forced into place to 
preserve the tatami property until either the top edge or the right edge is reached. Once again, we have two cases.
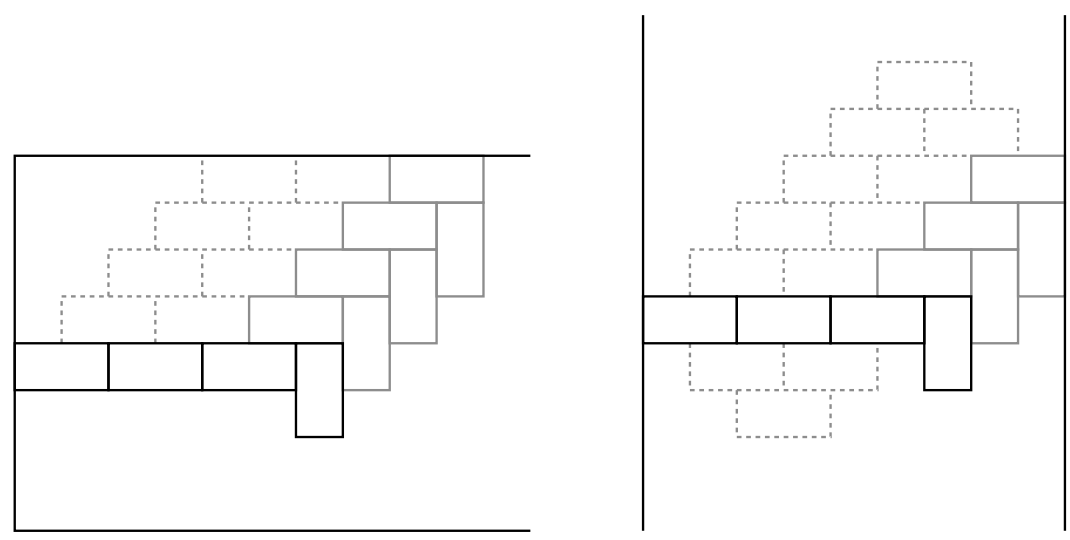

Figure 6: Forced tiles to preserve the tatami property due to a row with a horizontal tile on the left edge and at least one vertical tile.

case 2-1 If the top edge is reached, then, as shown by the grey dashed tiles in the left hand diagram of Figure 6, further forcing of tiles to preserve the tatami property yields an upper left corner that, by Theorem 2.1, we cannot tile.

case 2-2 If the right edge is reached, then, as shown in the right hand diagram of Figure 6, further forcing of tiles to preserve the tatami property yields a parallelogram of horizontal tiles as shown in Figure 7. This parallelogram consists of $n$ columns and $n-1$ rows since each column corresponds to exactly one row, excepting the two columns containing the top tile, which together correspond to one row. So $n-1 \leqslant m$ and since $n \geqslant m$ by definition, either $n-1=m-1$ or $n-1=m$ and in either case the parallelogram must touch either the top or bottom edge of the rectangle (or both). This leaves at least two triangular regions that, by Theorem 2.1, cannot be tiled, as shown in Figure 8.

Since we reached a contradiction in both cases, there cannot be a horizontal tile touching the left edge of the rectangle that is not either in the top or bottom row.

Theorem 2.3. If $m$ is odd and $1 \leqslant m \leqslant n$, a tatami tiling of an $m$ by $n$ rectangle consists of some combination of tatami tilings of $m$ by $m-1$ and $m$ by $m+1$ rectangles.

Proof. First note that when $m$ is odd, $n$ must be even for a tatami tiling (or any tiling with 1 by 2 tiles for that matter) to exist. When $m=1$ (and $n$ is even), the theorem is trivially true, as there is only one possible tatami tiling which consists entirely of horizontal tiles - tilings of 1 by 2 rectangles.

By Theorem 2.2, a tatami tiling of an $m$ by $n$ rectangle where $m$ is odd and $3 \leqslant m \leqslant n$ must have its leftmost column filled with vertical tiles except for either the top or bottom row. Assume, without loss of generality, that the bottom row contains the horizontal tile and the rest of the first column is filled with vertical tiles. Then, as shown by the 


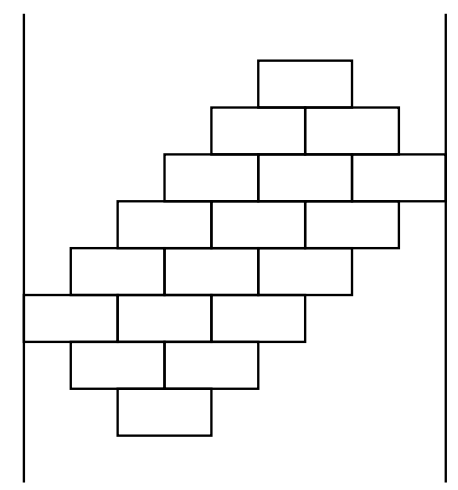

Figure 7: Parallelogram of horizontal tiles resulting from a row with a horizontal tile on the left edge and at least one vertical tile.
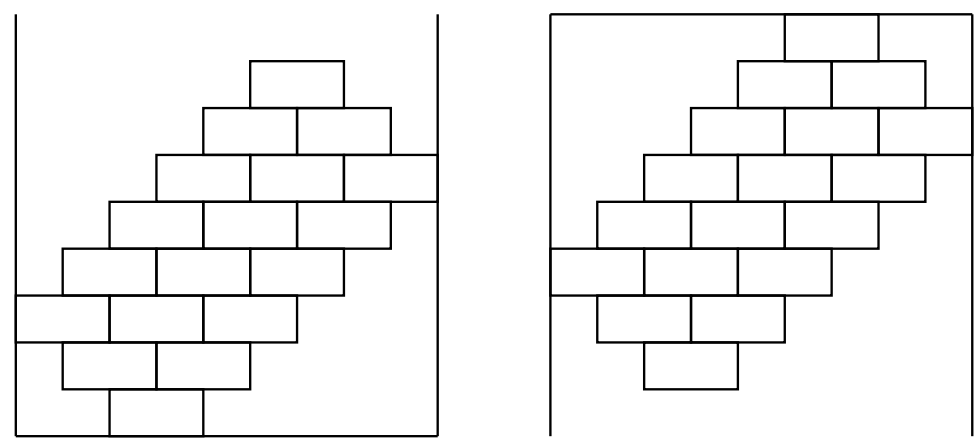

Figure 8: Parallelogram of horizontal tiles must touch either the top or bottom row (or both).

grey tiles in Figure 9, many tiles are forced into position in order to preserve the tatami property (when $m=3$ no tiles are forced but the tiles are already in position for the next step).

The leftmost pair of unoccupied squares can be filled in two ways: with a single vertical tile or with a pair of horizontal tiles. Once we fill this pair of squares, more tiles are forced into position to preserve the tatami property as shown in Figure 10 and we have filled either an $m$ by $m-1$ or $m$ by $m+1$ portion of our rectangle. Now, to preserve the tatami property, the leftmost column of the unfilled portion of our rectangle must be filled with vertical tiles except for the topmost square, which must contain a horizontal tile, and the same forcing process begins again. Thus the entire rectangle must be filled with some combination of $m$ by $m-1$ and $m$ by $m+1$ rectangles tiled as described.

Theorem 2.4. If $m$ is even and $4 \leqslant m \leqslant n$, a tatami tiling of an $m$ by $n$ rectangle consists of some combination of tatami tilings of $m$ by 1 rectangles, $m$ by $m-2$ rectangles and $m$ by $m$ rectangles such that the $m$ by 1 tilings appear between any two of the $m$ by $m-2$ and $m$ by $m$ tilings (and possibly at the left edge and/or the right edge of the rectangle). 


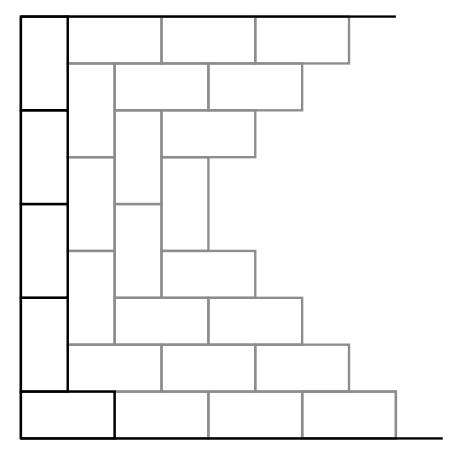

Figure 9: Forced tiles to preserve the tatami property due to $m$ being odd.
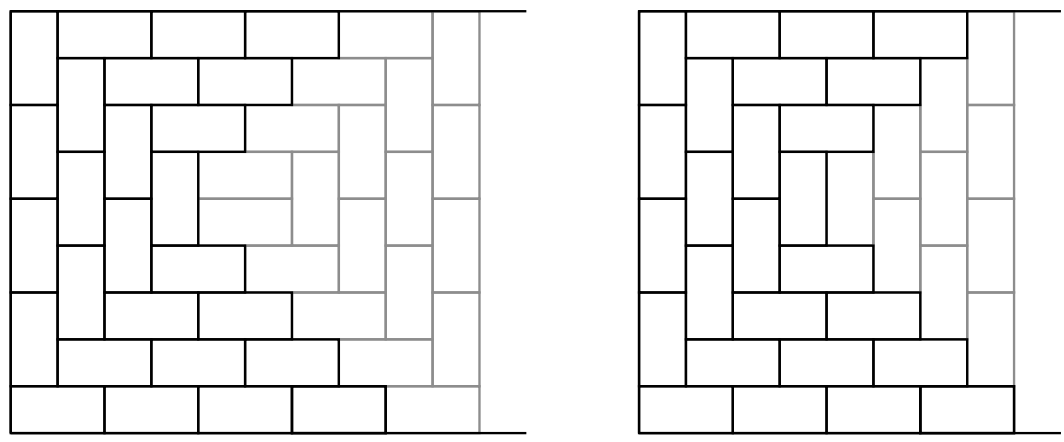

Figure 10: Filling an $m$ by $m+1$ or $m$ by $m-1$ portion of the rectangle.

Proof. By Theorem 2.2, a tatami tiling of an $m$ by $n$ rectangle where $m$ is even and $1 \leqslant m \leqslant n$ must either have its leftmost column filled with vertical tiles or have the top and bottom rows of its leftmost column filled with horizontal tiles and the rest of the leftmost column filled with vertical tiles, as shown in Figure 11. If the leftmost column is filled with vertical tiles, we have filled an $m$ by 1 portion of our rectangle and the next column must have horizontal tiles in the top and bottom row and otherwise be filled with vertical tiles.

Otherwise many tiles are forced into position in order to preserve the tatami property, as shown by the grey tiles in the right hand diagram of Figure 11. Now, as with the odd case, the leftmost pair of unoccupied squares can be filled in two ways: with a single vertical tile, or with a pair of horizontal tiles. Once we fill this pair of squares, more tiles are forced into position to preserve the tatami property as shown in Figure 12 and we have filled either an $m$ by $m-2$ or $m$ by $m$ portion of our rectangle. Now, to preserve the tatami property, the leftmost column of the unfilled portion of our rectangle must be completely filled with vertical tiles - in other words, it must contain an $m$ by 1 tiling. Again to preserve the tatami property, the column following an $m$ by 1 tiling must be filled with vertical tiles except for its top and bottom row which must contain horizontal tiles and the forcing process described above begins again. Thus the entire rectangle must be filled with some combination of $m$ by $m-2$ and $m$ by $m$ rectangles tiled as described and alternating with $m$ by 1 tiled rectangles. 

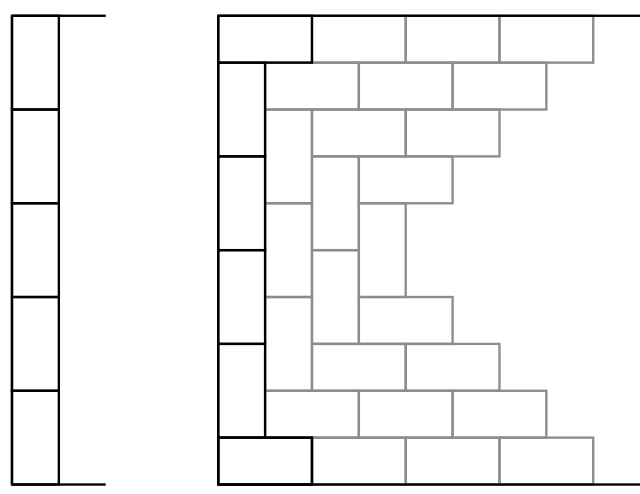

Figure 11: Possible tatami tilings of the leftmost column of an $m$ by $n$ rectangle when $n$ is even.
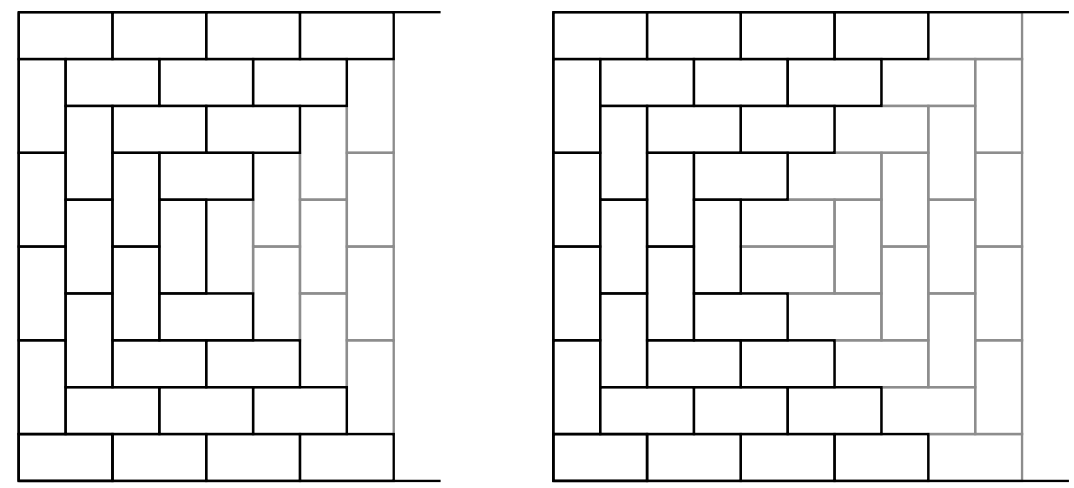

Figure 12: Filling an $m$ by $m-2$ or $m$ by $m$ portion of the rectangle.

\subsection{Compositions}

Hickerson's decomposition implies that there is a correspondence between certain compositions of $n$ and width $n$ tatami tilings. For $m=1$, the number $T(1, n)$ is the same as the number of compositions of $n$ into parts equal to 2 (which is 1 if $n$ is even and 0 otherwise). For $m=2$, the number $T(2, n)$ is the same as the number of compositions of $n$ into parts which are 1 or 2 , and in which no consecutive 2's occur. For $m>1$ odd, the number $T(m, n)$ is twice the number of compositions of $n$ into parts which are $m-1$ or $m+1$. For $m>2$ even, the number $T(m, n)$ is the same as the number of compositions of $n$ into parts which are 1 or $m-2$ or $m$, with the restriction that every other part is a 1. For example, the compositions of 22 corresponding to the tilings shown in Figure 2 are $2+1+1+2+1+1+1+2+1+2+1+1+1+1+1+2+1$ and $2+2+4+4+2+4+4$ and $2+1+4+1+2+1+2+1+4+1+2+1$. 


\section{$3 \quad$ Generating Functions}

Now that we know the limitations on the structures of tatami tilings with $1 \leqslant m \leqslant n$ we can begin to count them. We will use a similar approach to that used by Graham, et al, in Section 7.1 of Concrete Mathematics [2].

Let $T_{m}$ be the set of all tatami tilings of height $m$ and $T_{m}(z)$ be the ordinary generating function of these tilings where the coefficient of $z^{n}$ is the number of tilings of a rectangle with height $m$ and width $n$. Also, let $T_{m}(x, y)$ be the bivariate genrating function of the number of tatami tilings where the coefficient of $y^{v} x^{h}$ is the number of tilings with $v$ vertical and $h$ horizontal tiles.

The cases where $m=0$ and $m=1$ are trivial, with $T_{0}(z)=1$ and $T_{1}(z)=1 /\left(1-z^{2}\right)$.

\subsection{Counting Tilings when $m=2$}

When $m=2$, let $A$ be the formal sum of all height 2 tatami tilings that are either empty (denoted by $\mid$ ) or start with a vertical tile:

$$
A=\mid+\square A+\square A \text {. }
$$

Solving for $A$ gives:

$$
A=\frac{1}{1-1-\square}
$$

Now, let $T_{2}$ be all the 2 by $n$ tatami tilings and express $T_{2}$ in terms of $A$ :

$$
\begin{aligned}
T_{2} & =(\mid+\boxminus) A \\
& =(\mid+\boxminus) \frac{\mid}{\mid-\square-\square} .
\end{aligned}
$$

Since we want to know the number of tilings of a 2 by $n$ rectangle for $n \geqslant 0$, we can substitute $1\left(=z^{0}\right)$ for $\mid$, and $z^{n}$ for $\square, \square$, and $\square$ where $n$ is the width of each tiling (1, 2 , and 3 respectively):

$$
T_{2}(z)=\frac{1+z^{2}}{1-z-z^{3}}=1+z+2 z^{2}+3 z^{3}+4 z^{4}+6 z^{5}+9 z^{6}+13 z^{7}+19 z^{8}+28 z^{9}+\cdots
$$

which is the generating function for $T(2, n)$ for all values of $n$.

Substituting $y$ for each vertical tile and $x$ for each horizontal tile in (2), we obtain

$$
T_{2}(x, y)=\frac{1+x^{2}}{1-y\left(1+x^{2}\right)}=\sum_{k \geqslant 0} y^{k}\left(1+x^{2}\right)^{k+1}=\sum_{j \geqslant 0, k \geqslant 0}\left(\begin{array}{c}
k+1 \\
j
\end{array}\right) y^{k} x^{2 j}
$$

which proves the following theorem. 
Theorem 3.1. The number of tatami tilings of height 2 with $k$ vertical tiles and $2 j$ horizontal tiles is

$$
\left(\begin{array}{c}
k+1 \\
j
\end{array}\right)
$$

Corollary 3.2. The number of $2 \times n$ tatami tilings is

$$
\sum_{j, k \geqslant 0}\left(\begin{array}{c}
k+1 \\
j
\end{array}\right) \llbracket k+2 j=n \rrbracket=\sum_{j \geqslant 0}\left(\begin{array}{c}
n-2 j+1 \\
j
\end{array}\right) .
$$

\subsection{Counting Tilings when $m$ is Odd}

When $m$ is odd and $3 \leqslant m \leqslant n$, Theorem 2.3 showed that a tatami tiling of an $m$ by $n$ rectangle consists of some combination of tatami tilings of $m$ by $m-1$ and $m$ by $m+1$ rectangles. Note that tatami tilings of both the $m$ by $m-1$ rectangles and $m$ by $m+1$ rectangles must have either the top or bottom row (and not both) entirely filled with horizontal tiles. Thus we can effectively distinguish four possibilities for the tiling components of our $m$ by $n$ rectangle which we will denote as follows:

- $\boxminus: m$ by $m-1$ tiling with the top row filled with horizontal tiles,

- $\square$ : $m$ by $m+1$ tiling with the top row filled with horizontal tiles,

- $\boxminus$ : $m$ by $m-1$ tiling with the bottom row filled with horizontal tiles, and

- $\square$ : $m$ by $m+1$ tiling with the bottom row filled with horizontal tiles.

Let $A$ be the formal sum of the height $m$ tilings that are either empty or begin with a horizontal tile in the top row and $B$ be the formal sum of the height $m$ tilings that are either empty or begin with a horizontal tile in the bottom row.

$$
\begin{aligned}
& A=1+\square+\square+\square \square+\square \square+\square \square+\square \square+\cdots \\
& B=1+\square+\square+\square \square+\square \square+\square \square+\square \square+\cdots
\end{aligned}
$$

Define $\alpha$ and $\beta$ as shown below.

$$
\alpha=(\boxminus+\square) \text { and } \beta=(\boxminus+\square) \text {. }
$$

Then $A$ and $B$ can be expressed in terms of each other:

$$
A=\mid+\alpha B \text { and } B=\mid+\beta A .
$$

We can substitute for $B$ to get $A=\mid+\alpha+\alpha \beta A$. Solving for $A$ and substituting the result into $B$ we obtain

$$
A=(\mid-\alpha \beta)^{-1}(\mid+\alpha) \quad \text { and } \quad B=\mid+\beta(\mid-\alpha \beta)^{-1}(\mid+\alpha)
$$




\begin{tabular}{c|c|c|} 
dimensions & horizontal & vertical \\
\hline$m \times(m+1), m$ odd & $\ell^{2}$ & $\ell(\ell-1)$ \\
\hline$m \times(m-1), m$ odd & $(\ell-1)^{2}$ & $\ell(\ell-1)$ \\
\hline$m \times(m-2), m$ even & $\ell(\ell-1)$ & $\ell(\ell-1)$ \\
\hline$m \times m, m$ even & $\ell(\ell+1)$ & $\ell(\ell-1)$ \\
\hline$m \times 1, m$ even & 0 & $\ell$ \\
\hline
\end{tabular}

Table 1: Number of horizontal and vertical tiles (with $\ell=\lceil m / 2\rceil$ ).

Thus

$$
\begin{aligned}
T_{m} & =A+B-\mid \\
& =(\mid-\alpha \beta)^{-1}(\mid+\alpha)+\left|+\beta(\mid-\alpha \beta)^{-1}(\mid+\alpha)-\right| \\
& =(\mid+\beta)(\mid-\alpha \beta)^{-1}(\mid+\alpha) \\
& =(\mid+\square+\square)(\mid-(\square+\square)(\square+\square))^{-1}(\mid+\square+\square)
\end{aligned}
$$

Since we want to know the number of tilings of an $m$ by $n$ rectangle, we can substitute:

- 1 for $\mid$,

- $z^{m-1}$ for $\boxminus$ and $\boxminus$, and

- $z^{m+1}$ for $\longmapsto$ and $\square$

into (3) to get a generating function for the number of $m$ by $n$ tilings with $3 \leqslant m \leqslant n$ and $m$ odd:

$$
\begin{aligned}
T_{m}(z) & =\frac{\left(1+z^{m-1}+z^{m+1}\right)^{2}}{1-\left(z^{m-1}+z^{m+1}\right)^{2}} \\
& =\frac{1+z^{m-1}+z^{m+1}}{1-z^{m-1}-z^{m+1}} .
\end{aligned}
$$

The coefficient of $z^{n}$ in the power series generated by (4) with an odd value of $m$ and when $3 \leqslant m \leqslant n$ is $T(m, n)$.

We now try to count the tatami tilings according to the number of horizontal and vertical tiles. Let $\ell=(m+1) / 2$. Table 1 shows the number of horizontal and vertical tiles in the $m \times(m+1)$ rectangle and in the $m \times(m-1)$ rectangle.

We then obtain from (4) and Table 1 that

$$
T_{m}(x, y)=\frac{1+y^{\ell(\ell-1)} x^{\ell^{2}}+y^{\ell(\ell-1)} x^{(\ell-1)^{2}}}{1-y^{\ell(\ell-1)} x^{\ell^{2}}-y^{\ell(\ell-1)} x^{(\ell-1)^{2}}}=\frac{1+y^{\ell(\ell-1)} x^{(\ell-1)^{2}}\left(1+x^{2 \ell-1}\right)}{1-y^{\ell(\ell-1)} x^{(\ell-1)^{2}}\left(1+x^{2 \ell-1}\right)}
$$

We now count the number of tilings by the number of horizontal and vertical tiles. 
Theorem 3.3. For $m$ odd, the number of tatami tilings with $k\left(\ell^{2}-\ell\right)=k\left(m^{2}-1\right) / 4$ vertical and $k(\ell-1)^{2}+j(2 \ell-1)=k(m-1)^{2} / 4+j m$ horizontal tiles is

$$
2\left(\begin{array}{c}
k \\
j
\end{array}\right) .
$$

Proof. First note that $(1+a) /(1-a)=1+2 a+2 a^{2}+\cdots$ is the form of (5), so that, except for the constant term, the coefficient is twice what it is for $1 /(1-a)$. Thus we expand as follows

$$
\begin{aligned}
\frac{1}{1-y^{\ell^{2}-\ell} x^{(\ell-1)^{2}}\left(1+x^{2 \ell-1}\right)} & =\sum_{k \geqslant 0}\left(y^{\ell^{2}-\ell} x^{(\ell-1)^{2}}\left(1+x^{2 \ell-1}\right)\right)^{k} \\
& =\sum_{k \geqslant 0} y^{k\left(\ell^{2}-\ell\right)} x^{k(\ell-1)^{2}} \sum_{j \geqslant 0}\left(\begin{array}{l}
k \\
j
\end{array}\right) x^{j(2 \ell-1)} \\
& =\sum_{k \geqslant 0, j \geqslant 0}\left(\begin{array}{l}
k \\
j
\end{array}\right) y^{k\left(\ell^{2}-\ell\right)} x^{k(\ell-1)^{2}+j(2 \ell-1)} .
\end{aligned}
$$

If $\alpha$ is not an integer, then we adopt the convention that the binomial coefficient $\left(\begin{array}{c}\alpha \\ j\end{array}\right)$ is zero.

Corollary 3.4. For $m$ odd and $3 \leqslant m \leqslant n$, the number of $m \times n$ tatami tilings is

$$
2 \sum_{j, k \geqslant 0}\left(\begin{array}{l}
k \\
j
\end{array}\right) \llbracket n=k(m-1)+2 j \rrbracket=2 \sum_{j \geqslant 0}\left(\begin{array}{c}
(n-2 j) /(m-1) \\
j
\end{array}\right)
$$

Proof. The total number of tiles in the statement of Theorem 3.3 is

$$
k\left(m^{2}-1\right) / 4+k(m-1)^{2} / 4+j m=k m(m-1) / 2+j m .
$$

On the other hand, the total number of tiles in a $n \times m$ tatami tiling is $n m / 2$. Thus we have $n=k(m-1)+2 j$.

\subsection{Counting Tilings when $m$ is Even}

When $m$ is even and $4 \leqslant m \leqslant n$, Theorem 2.4 showed that a tatami tiling of an $m$ by $n$ rectangle consists of some combination of tatami tilings of $m$ by $m-2$ and $m$ by $m$ rectangles with $m$ by 1 rectangles in between them and possibly in the first or last column or both. Note that tatami tilings of both the $m$ by $m-2$ rectangles and $m$ by $m$ rectangles must have both the top and bottom row entirely filled with horizontal tiles. Thus we denote the three possibilities for the tiling components of our $m$ by $n$ rectangle as follows: 
- $\theta: m$ by 1 tiling (all vertical tiles),

- 田: $m$ by $m-2$ tiling, and

- $\boxminus$ : $m$ by $m$ tiling.

Let $A$ be the formal sum of the height $m$ tilings which do not begin with an $m$ by 1 tiling and $B$ be the formal sum of the height $m$ tilings which do begin with an $m$ by 1 tiling:

$$
\begin{aligned}
& A=1+\mathbb{D}+\vec{H}+\mathbb{I}+\mathrm{H}
\end{aligned}
$$

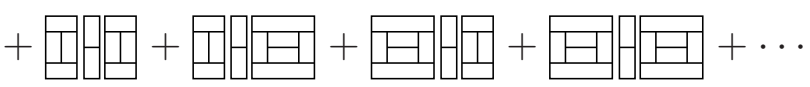

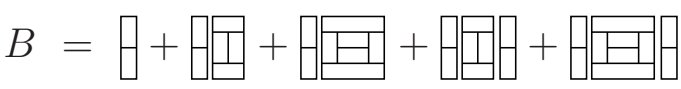

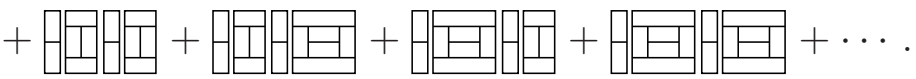

Define $\alpha$ and $\beta$ as shown below.

$$
\alpha=(\mathbb{E}+\ddot{E}) \text { and } \beta=\text {. }
$$

Then $A$ and $B$ can be expressed in terms of each other:

$$
A=\mid+\alpha(\mid+B) \text { and } B=\beta A .
$$

We can substitute for $B$ to get $A=\mid+\alpha+\alpha \beta A$. Solving for $A$, substituting the result, and solving for $B$ we obtain

$$
A=(\mid-\alpha \beta)^{-1}(\mid+\alpha) \text { and } B=\beta(\mid-\alpha \beta)^{-1}(\mid+\alpha)
$$

Thus

$$
\begin{aligned}
& T_{m}=A+B \\
& =(\mid-\alpha \beta)^{-1}(\mid+\alpha)+\beta(\mid-\alpha \beta)^{-1}(\mid+\alpha) \\
& =(\mid+\beta)(\mid-\alpha \beta)^{-1}(\mid+\alpha)
\end{aligned}
$$

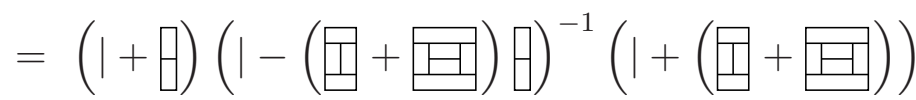

Since we want to know the number of tilings of an $m$ by $n$ rectangle, we can substitute:

- 1 for $\mid$,

- $z$ for 7 ,

- $z^{m-2}$ for 1 , 
- $z^{m}$ for $\mathrm{H}$

into (8) to get a generating function for the number of $m$ by $n$ tatami tilings with $4 \leqslant$ $m \leqslant n$ and $m$ even:

$$
\begin{aligned}
T_{m}(z) & =\frac{(1+z)\left(1+z^{m-2}+z^{m}\right)}{1-\left(z^{m-2}-z^{m}\right) z} \\
& =\frac{(1+z)\left(1+z^{m-2}+z^{m}\right)}{1-z^{m-1}-z^{m+1}} .
\end{aligned}
$$

The coefficient of $z^{n}$ in the power series generated by (9) with an even value of $m$ and when $4 \leqslant m \leqslant n$ is $T(m, n)$.

Referring to (9) and again to Table 1, the corresponding bivariate generating function is

$$
\begin{aligned}
T_{m}(x, y) & =\frac{\left(1+y^{\ell}\right)\left(1+y^{\ell(\ell-1)} x^{\ell(\ell-1)}+y^{\ell(\ell-1)} x^{\ell(\ell+1)}\right)}{1-y^{\ell^{2}} x^{\ell(\ell-1)}-y^{\ell^{2}} x^{\ell(\ell+1)}} \\
& =\frac{1+y^{\ell^{2}}\left(x^{\ell(\ell-1)}+x^{\ell(\ell+1)}\right)}{1-y^{\ell^{2}}\left(x^{\ell(\ell-1)}+x^{\ell(\ell+1)}\right)}+\frac{y^{\ell}\left(1+y^{\ell(\ell-2)}\left(x^{\ell(\ell-1)}+x^{\ell(\ell+1)}\right)\right)}{1-y^{\ell^{2}}\left(x^{\ell(\ell-1)}+x^{\ell(\ell+1)}\right)}
\end{aligned}
$$

Using the same sort of expansion as used in the proof of Theorem 3.3 on equation (10), we obtain that $1+T_{m}(x, y)$ is

$$
y^{\ell}\left(1+y^{\ell(\ell-2)}\left(x^{\ell(\ell-1)}+x^{\ell(\ell+1)}\right)\right) \sum_{j, k \geqslant 0}\left(\begin{array}{l}
k \\
j
\end{array}\right) y^{k \ell^{2}} x^{k \ell(\ell-1)+2 j \ell} .
$$

This expansion could be used to state a messy analogue of Theorem 3.3, which we omit. However, we do give an expression as a sum of binomial coefficients below.

Theorem 3.5. For $m$ even and $4 \leqslant m \leqslant n$, the number of $m \times n$ tatami tilings is

$$
\sum_{j \geqslant 0}\left(2\left(\begin{array}{c}
\frac{n-2 j}{m-1} \\
j
\end{array}\right)+\left(\begin{array}{c}
\frac{n-2 j-1}{m-1} \\
j
\end{array}\right)+\left(\begin{array}{c}
\frac{n-2 j-m+2}{m-1} \\
j
\end{array}\right)+\left(\begin{array}{c}
\frac{n-2 j-m}{m-1} \\
j
\end{array}\right)\right) .
$$

Proof. Note that the denominator of (9) can be expanded as

$$
\frac{1}{1-z^{m-1}-z^{m+1}}=\sum_{j, k \geqslant 0}\left(\begin{array}{l}
k \\
j
\end{array}\right) z^{k(m-1)+2 j},
$$

and that the numerator is

$$
(1+z)\left(1+z^{m-2}+z^{m}\right)=\left(1+z^{m-1}+z^{m+1}\right)+(z)+\left(z^{m-2}\right)+\left(z^{m}\right) .
$$

Thus in the four sums corresponding to the four parenthesized terms on the righthand side above, if $n$ is the exponent of $z$, then either: (a) $n=k(m-1)+2 j$, (b) $n=k(m-1)+2 j+1$, (c) $n=k(m-1)+2 j+m-2$, or (d) $n=k(m-1)+2 j+m$. Now solving for $k$ we get the four terms in the summation in the statement of the theorem. 


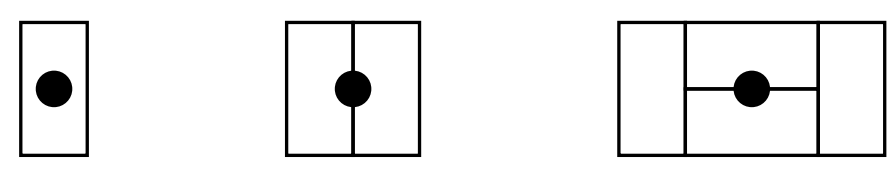

Figure 13: Center tiles in symmetric figurations for $m=2$.

\subsection{An Exposé of Knuth's Generating Function}

Recall Knuth's generating function (1). A simple calculation shows that when $m \geqslant 4$ is even,

$$
\left(1-z^{m-1}-z^{m+1}\right)\left(T_{m}(z)-K(z)\right)=(1+z)\left(1-z^{m-1}-z^{m+1}\right) .
$$

Note that the two generating functions differ in only in the constant coefficient and the coefficient of $z$. Thus Knuth's generating function is valid for even $m$ such that $4 \leqslant m \leqslant n$, since $T_{m}(z)$ is valid then. The question remains of whether it (and thus $T_{m}(z)$ ) also gives the correct coefficients when $n=m-1$ and/or $n=m-2$. For $m>6$, the coefficient of $z^{m-2}$ is 1 and for $m>4$ the coefficient of $z^{m-1}$ is 2 . The corresponding compositions are $m-2=(m-2)$ and $m-1=(1)+(m-2)=(m-2)+(1)$. However, there are two caveats; firstly, that when $m=4$, we have $T(4,2)=T(4,3)=4$ and secondly, that when $m=6$, we have $T(6,4)=3$. Thus $K(z)$ is correct for $6 \leqslant m-2 \leqslant n$, for $5 \leqslant m-1 \leqslant n$, and for $4 \leqslant m \leqslant n$ but not outside those ranges.

\section{Inequivalent Tilings}

Two tilings are congruent if there is some isometry in 3-space that maps one to the other. In this section we derive generating functions for the number of non-congruent tatami tilings. We use the terms non-congruent and inequivalent interchangeably.

We use $U_{m}(z)$ to denote the ordinary generating function of $U(m, n)$, the number of height $m$ width $n$ tilings that are symmetric about a vertical line through the center point $x$ of the tiling ( $x$ is denoted by a black circle in Figures 13, 14, and 15). The ordinary generating function of the number of inequivalent tilings, $I(m, n)$, is denoted $I_{m}(z)$. As in the previous sections, these generating functions are valid only for restricted values of $n$.

As with unrestricted tilings, the cases where $m=0$ and $m=1$ are trivial, with $I_{0}(z)=U_{0}(z)=T_{0}(z)=1$ and $I_{1}(z)=U_{1}(z)=T_{1}(z)=1 /\left(1-z^{2}\right)$.

\subsection{Height 2 tilings}

When $m=2$ there are three possibilities for the center of the tiling, as illustrated by Figure 13. Either $n$ is odd and $x$ is in the center of a single vertical tile, or $n$ is even, in which case one of the following two cases apply. If $n$ is even, then $x$ could lie between two vertical tiles, or it could lie between two horizontal tiles with two vertical tiles bordering 

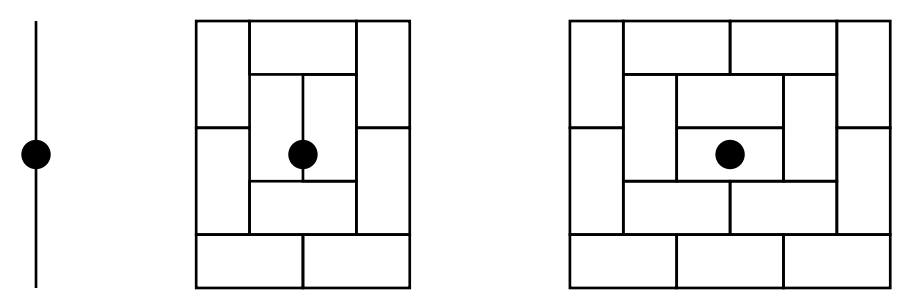

Figure 14: Center tiles in symmetric figurations for $m$ odd; illustrated for $m=5$.

them. Each of these has a tiling $\alpha$ abutting it on the left and $\alpha^{R}$ (the reversal of $\alpha$ ) on the right. Thus

$$
U_{2}(z)=1+\left(z+z^{2}+z^{4}\right) T_{2}\left(z^{2}\right)=\frac{1+z+z^{4}+z^{5}+z^{8}}{1-z^{2}-z^{6}}
$$

Therefore the generating function for the number of inequivalent tilings is

$$
I_{2}(z)=\frac{1}{2}\left(T_{2}(z)+U_{2}(z)-z^{2}\right)=\frac{1-z^{2}-z^{6}-z^{7}-z^{9}-z^{11}}{\left(1-z-z^{3}\right)\left(1-z^{2}-z^{6}\right)} .
$$

The $-z^{2}$ is to account for the fact that there is only one inequivalent 2 by 2 tiling, but it has an addition isometry, namely a 90 degree rotation. The generating functions (12) and (13) are accurate for all values of $n \geqslant 0$.

\subsection{Odd height tilings}

For $m$ odd, there appear to be three possibilities for the center tiles (up to a horizontal reflection) as illustrated in Figure 14, however the leftmost one is actually a rotational symmetry about the point $x$. Each of these latter tilings has a tiling $\alpha$ on the left and $\alpha^{\bar{R}}$ (the 180 degree rotation of $\alpha$ ) on the right. Each tiling of the other two types has a tiling $\alpha$ abutting it on the left and $\alpha^{R}$ on the right. For each of the three configurations of Figure 15, $\alpha$ is an unrestricted Tatami tiling of height $m$. As before, we let $U_{m}(z)$ be the generating function for the tilings symmetric about a vertical line, but we will also define $U_{m}^{R}(z)$ to be the generating function for the tilings with the rotational symmetry.

Hence

$$
\begin{aligned}
U_{m}(z) & =z^{m-1}+z^{m+1}+\left(1+z^{m-1}+z^{m+1}\right) T_{m}\left(z^{2}\right) \\
& =\frac{1+2\left(z^{m-1}+z^{m+1}\right)+z^{2 m-2}+z^{2 m+2}}{1-z^{2 m-2}-z^{2 m+2}}
\end{aligned}
$$

and

$$
U_{m}^{R}(z)=T_{m}\left(z^{2}\right)=\frac{1+z^{2 m-2}+z^{2 m+2}}{1-z^{2 m-2}-z^{2 m+2}}
$$



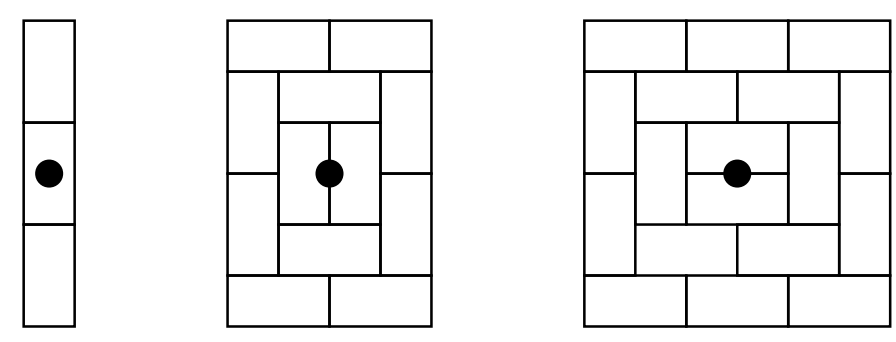

Figure 15: Center tiles in symmetric figurations for $m$ even; illustrated for $m=6$.

The number of inequivalent tatami tilings, $I(m, n)$, for $3 \leqslant m \leqslant n$ is given by the coefficients of

$$
\begin{aligned}
I_{m}(z) & =\frac{1}{4}\left(U_{m}(z)+U_{m}^{R}(z)+T_{m}(z)\right) \\
& =\frac{1}{4}\left(\frac{2\left(1+z^{m-1}+z^{m+1}+z^{2 m-2}+z^{2 m+2}\right)}{1-z^{2 m-2}-z^{2 m+2}}+\frac{1+z^{m-1}+z^{m+1}}{1-z^{m-1}-z^{m+1}}\right)
\end{aligned}
$$

When $m=3$ the number of tatami tilings has a nice expression in terms of the Fibonacci numbers; see OEIS [6] A001224, A060312, A068928, and A102526.

\subsection{Even height tilings}

For $m$ even, there are three possibilities for the center tiles, as illustrated in Figure 15; each of these has a tiling $\alpha$ abutting it on the left and $\alpha^{R}$ on the right. For the leftmost configuration of Figure 15, $\alpha$ cannot end in a column of vertical tiles; in the other two configurations $\alpha$ must end in a column of vertical tiles. The generating functions for these restricted tilings were essentially determined in (6) and (7), namely

$$
A_{m}(z)=\frac{1+z^{m-2}+z^{m}}{1-z^{m-1}-z^{m+1}} \quad \text { and } B_{m}(z)=z A_{m}(z)=\frac{z+z^{m-1}+z^{m+1}}{1-z^{m-1}-z^{m+1}} .
$$

Thus

$$
U_{m}(z)=z A_{m}\left(z^{2}\right)+\left(z^{m-2}+z^{m}\right) B_{m}\left(z^{2}\right)=\frac{\left(z+z^{m}+z^{m+2}\right)\left(1+z^{2 m-4}+z^{2 m}\right)}{1-z^{2 m-2}-z^{2 m+2}} .
$$

The number of inequivalent tatami tilings for $4 \leqslant m \leqslant n$ is given by the coefficients of

$$
\begin{aligned}
I_{m}(z) & =\frac{1}{2}\left(U_{m}(z)+T_{m}(z)-z^{m}\right) \\
& =\frac{1}{2}\left(\frac{\left(z+z^{m}+z^{m+2}\right)\left(1+z^{2 m-4}+z^{2 m}\right)}{1-z^{2 m-2}-z^{2 m+2}}+\frac{(1+z)\left(1+z^{m-2}+z^{m}\right)}{1-z^{m-1}-z^{m+1}}-z^{m}\right) .
\end{aligned}
$$

Like the $m=2$ case, the $-z^{m}$ is to account for the fact that the single inequivalent $m$ by $m$ tiling has a 90 degree rotational symmetry and thus is accounted for twice in $T_{m}(z)$. 


\section{Final Comments}

The following equations summarize the generating functions for $T(m, n)$, the number of unrestricted tatami tilings,

$$
T_{m}(z)=\sum_{n \geqslant 0} T(m, n) z^{n}= \begin{cases}1 & \text { for } m=0 \\ \frac{1}{1-z^{2}} & \text { for } m=1 \\ \frac{1+z^{2}}{1-z-z^{3}} & \text { for } m=2 \\ \frac{1+z^{m-1}+z^{m+1}}{1-z^{m-1}-z^{m+1}} & \text { for } m \text { odd }, 3 \leqslant m \leqslant n \\ \frac{(1+z)\left(1+z^{m-2}+z^{m}\right)}{1-z^{m-1}-z^{m+1}} & \text { for } m \text { even, } 4 \leqslant m \leqslant n\end{cases}
$$

$U(m, n)$, the number of vertically symmetrical tatami tilings,

$$
U_{m}(z)=\sum_{n \geqslant 0} U(m, n) z^{n}= \begin{cases}1 & \text { for } m=0 \\ \frac{1}{1-z^{2}} & \text { for } m=1 \\ \frac{1+z+z^{4}+z^{5}+z^{8}}{1-z^{2}-z^{6}} & \text { for } m=2 \\ \frac{1+2\left(z^{m-1}+z^{m+1}\right)+z^{2 m-2}+z^{2 m+2}}{1-z^{2 m-2}-z^{2 m+2}} & \text { for } m \text { odd, } 3 \leqslant m \leqslant n \\ \frac{\left(z+z^{m}+z^{m+2}\right)\left(1+z^{2 m-4}+z^{2 m}\right)}{1-z^{2 m-2}-z^{2 m+2}} & \text { for } m \text { even, } 4 \leqslant m \leqslant n,\end{cases}
$$

and $I(m, n)$, the number of inequivalent tatami tilings,

$$
\begin{aligned}
& I_{m}(z)= \sum_{n \geqslant 0} I(m, n) z^{n} \\
&= \begin{cases}1 & \text { for } m=0 \\
\frac{1}{1-z^{2}} & \text { for } m=1 \\
\frac{1-z^{2}-z^{6}-z^{7}-z^{9}-z^{11}}{\left(1-z-z^{3}\right)\left(1-z^{2}-z^{6}\right)} & \text { for } m=2 \\
\frac{1}{4}\left(\frac{2\left(1+z^{m-1}+z^{m+1}+z^{2 m-2}+z^{2 m+2}\right)}{1-z^{2 m-2}-z^{2 m+2}}+T_{m}(z)\right) & \text { for } m \text { odd, } 3 \leqslant m \leqslant n \\
\frac{1}{2}\left(\frac{\left(z+z^{m}+z^{m+2}\right)\left(1+z^{2 m-4}+z^{2 m}\right)}{1-z^{2 m-2}-z^{2 m+2}}+T_{m}(z)-z^{m}\right) & \text { for } m \text { even, } 4 \leqslant m \leqslant n .\end{cases}
\end{aligned}
$$

Tables 2, 3, and 4, respectively, show the numbers produced by these generating functions for $1 \leqslant m, n \leqslant 16$.

\section{Acknowledgements}

We wish to thank Dean Hickerson for allowing us to use and expand upon the combinatorial decomposition that he first presented in [3], and Don Knuth for depositing one hexadecimal dollar into the second author's account at the The Bank of San Serriffe after he was sent an earlier draft of this paper. 


\begin{tabular}{r|rrrrrrrrrrrrrrrr} 
& 1 & 2 & 3 & 4 & 5 & 6 & 7 & 8 & 9 & 10 & 11 & 12 & 13 & 14 & 15 & 16 \\
\hline 1 & 0 & 1 & 0 & 1 & 0 & 1 & 0 & 1 & 0 & 1 & 0 & 1 & 0 & 1 & 0 & 1 \\
2 & 1 & 2 & 3 & 4 & 6 & 9 & 13 & 19 & 28 & 41 & 60 & 88 & 129 & 189 & 277 & 406 \\
3 & 0 & 3 & 0 & 4 & 0 & 6 & 0 & 10 & 0 & 16 & 0 & 26 & 0 & 42 & 0 & 68 \\
4 & 1 & 4 & 4 & 2 & 3 & 3 & 3 & 5 & 5 & 6 & 8 & 8 & 11 & 13 & 14 & 19 \\
5 & 0 & 6 & 0 & 3 & 0 & 2 & 0 & 2 & 0 & 4 & 0 & 4 & 0 & 6 & 0 & 8 \\
6 & 1 & 9 & 6 & 3 & 2 & 2 & 2 & 1 & 1 & 2 & 3 & 4 & 3 & 3 & 3 & 4 \\
7 & 0 & 13 & 0 & 3 & 0 & 2 & 0 & 2 & 0 & 0 & 0 & 2 & 0 & 4 & 0 & 2 \\
8 & 1 & 19 & 10 & 5 & 2 & 1 & 2 & 2 & 2 & 1 & 0 & 0 & 1 & 2 & 3 & 4 \\
9 & 0 & 28 & 0 & 5 & 0 & 1 & 0 & 2 & 0 & 2 & 0 & 0 & 0 & 0 & 0 & 2 \\
10 & 1 & 41 & 16 & 6 & 4 & 2 & 0 & 1 & 2 & 2 & 2 & 1 & 0 & 0 & 0 & 0 \\
11 & 0 & 60 & 0 & 8 & 0 & 3 & 0 & 0 & 0 & 2 & 0 & 2 & 0 & 0 & 0 & 0 \\
12 & 1 & 88 & 26 & 8 & 4 & 4 & 2 & 0 & 0 & 1 & 2 & 2 & 2 & 1 & 0 & 0 \\
13 & 0 & 129 & 0 & 11 & 0 & 3 & 0 & 1 & 0 & 0 & 0 & 2 & 0 & 2 & 0 & 0 \\
14 & 1 & 189 & 42 & 13 & 6 & 3 & 4 & 2 & 0 & 0 & 0 & 1 & 2 & 2 & 2 & 1 \\
15 & 0 & 277 & 0 & 14 & 0 & 3 & 0 & 3 & 0 & 0 & 0 & 0 & 0 & 2 & 0 & 2 \\
16 & 1 & 406 & 68 & 19 & 8 & 4 & 2 & 4 & 2 & 0 & 0 & 0 & 0 & 1 & 2 & 2
\end{tabular}

Table 2: The numbers $T(m, n)$ for $1 \leqslant m, n \leqslant 16$.

\begin{tabular}{r|rrrrrrrrrrrrrrrr} 
& 1 & 2 & 3 & 4 & 5 & 6 & 7 & 8 & 9 & 10 & 11 & 12 & 13 & 14 & 15 & 16 \\
\hline 1 & 0 & 1 & 0 & 1 & 0 & 1 & 0 & 1 & 0 & 1 & 0 & 1 & 0 & 1 & 0 & 1 \\
2 & 1 & 1 & 1 & 2 & 2 & 3 & 3 & 5 & 4 & 7 & 6 & 10 & 9 & 15 & 13 & 22 \\
3 & 0 & 2 & 0 & 4 & 0 & 2 & 0 & 6 & 0 & 4 & 0 & 10 & 0 & 6 & 0 & 16 \\
4 & 1 & 0 & 0 & 1 & 1 & 1 & 1 & 1 & 1 & 2 & 2 & 2 & 1 & 3 & 2 & 3 \\
5 & 0 & 0 & 0 & 2 & 0 & 2 & 0 & 2 & 0 & 0 & 0 & 4 & 0 & 2 & 0 & 4 \\
6 & 1 & 0 & 0 & 0 & 0 & 1 & 0 & 1 & 1 & 0 & 1 & 0 & 1 & 1 & 1 & 2 \\
7 & 0 & 0 & 0 & 0 & 0 & 2 & 0 & 2 & 0 & 0 & 0 & 2 & 0 & 0 & 0 & 2 \\
8 & 1 & 0 & 0 & 0 & 0 & 0 & 0 & 1 & 0 & 1 & 0 & 0 & 1 & 0 & 1 & 0 \\
9 & 0 & 0 & 0 & 0 & 0 & 0 & 0 & 2 & 0 & 2 & 0 & 0 & 0 & 0 & 0 & 2 \\
10 & 1 & 0 & 0 & 0 & 0 & 0 & 0 & 0 & 0 & 1 & 0 & 1 & 0 & 0 & 0 & 0 \\
11 & 0 & 0 & 0 & 0 & 0 & 0 & 0 & 0 & 0 & 2 & 0 & 2 & 0 & 0 & 0 & 0 \\
12 & 1 & 0 & 0 & 0 & 0 & 0 & 0 & 0 & 0 & 0 & 0 & 1 & 0 & 1 & 0 & 0 \\
13 & 0 & 0 & 0 & 0 & 0 & 0 & 0 & 0 & 0 & 0 & 0 & 2 & 0 & 2 & 0 & 0 \\
14 & 1 & 0 & 0 & 0 & 0 & 0 & 0 & 0 & 0 & 0 & 0 & 0 & 0 & 1 & 0 & 1 \\
15 & 0 & 0 & 0 & 0 & 0 & 0 & 0 & 0 & 0 & 0 & 0 & 0 & 0 & 2 & 0 & 2 \\
16 & 1 & 0 & 0 & 0 & 0 & 0 & 0 & 0 & 0 & 0 & 0 & 0 & 0 & 0 & 0 & 1
\end{tabular}

Table 3: The numbers $U(m, n)$ for $1 \leqslant m, n \leqslant 16$. 


\begin{tabular}{r|rrrrrrrrrrrrrrrr} 
& 1 & 2 & 3 & 4 & 5 & 6 & 7 & 8 & 9 & 10 & 11 & 12 & 13 & 14 & 15 & 16 \\
\hline 1 & 0 & 1 & 0 & 1 & 0 & 1 & 0 & 1 & 0 & 1 & 0 & 1 & 0 & 1 & 0 & 1 \\
2 & 1 & 1 & 2 & 3 & 4 & 6 & 8 & 12 & 16 & 24 & 33 & 49 & 69 & 102 & 145 & 214 \\
3 & 0 & 2 & 0 & 2 & 0 & 2 & 0 & 4 & 0 & 5 & 0 & 9 & 0 & 12 & 0 & 21 \\
4 & 1 & 3 & 2 & 1 & 2 & 2 & 2 & 3 & 3 & 4 & 5 & 5 & 6 & 8 & 8 & 11 \\
5 & 0 & 4 & 0 & 2 & 0 & 1 & 0 & 1 & 0 & 1 & 0 & 2 & 0 & 2 & 0 & 3 \\
6 & 1 & 6 & 2 & 2 & 1 & 1 & 1 & 1 & 1 & 1 & 2 & 2 & 2 & 2 & 2 & 3 \\
7 & 0 & 8 & 0 & 2 & 0 & 1 & 0 & 1 & 0 & 0 & 0 & 1 & 0 & 1 & 0 & 1 \\
8 & 1 & 12 & 4 & 3 & 1 & 1 & 1 & 1 & 1 & 1 & 0 & 0 & 1 & 1 & 2 & 2 \\
9 & 0 & 16 & 0 & 3 & 0 & 1 & 0 & 1 & 0 & 1 & 0 & 0 & 0 & 0 & 0 & 1 \\
10 & 1 & 24 & 5 & 4 & 1 & 1 & 0 & 1 & 1 & 1 & 1 & 1 & 0 & 0 & 0 & 0 \\
11 & 0 & 33 & 0 & 5 & 0 & 2 & 0 & 0 & 0 & 1 & 0 & 1 & 0 & 0 & 0 & 0 \\
12 & 1 & 49 & 9 & 5 & 2 & 2 & 1 & 0 & 0 & 1 & 1 & 1 & 1 & 1 & 0 & 0 \\
13 & 0 & 69 & 0 & 6 & 0 & 2 & 0 & 1 & 0 & 0 & 0 & 1 & 0 & 1 & 0 & 0 \\
14 & 1 & 102 & 12 & 8 & 2 & 2 & 1 & 1 & 0 & 0 & 0 & 1 & 1 & 1 & 1 & 1 \\
15 & 0 & 145 & 0 & 8 & 0 & 2 & 0 & 2 & 0 & 0 & 0 & 0 & 0 & 1 & 0 & 1 \\
16 & 1 & 214 & 21 & 11 & 3 & 3 & 1 & 2 & 1 & 0 & 0 & 0 & 0 & 1 & 1 & 1
\end{tabular}

Table 4: The numbers $I(m, n)$ for $1 \leqslant m, n \leqslant 16$.

\section{References}

[1] Martin Aigner. A Course in Enumeration. Springer, 2007.

[2] Ronald L. Graham, Donald E. Knuth, and Oren Patashnik. Concrete Mathematics. Addison-Wesley, USA, 2nd edition, 1994.

[3] Dean Hickerson. Filling rectangular rooms with tatami mats. http: //www.research . att.com/ njas/sequences/a068920.txt, March 2002.

[4] Donald E. Knuth. The Art of Computer Programming, volume 4, Fascicle 1: Bitwise Tricks and Techniques; Binary Decision Diagrams. Addison-Wesley, USA, 2009.

[5] Ronald C. Read. The dimer problem for narrow rectangular arrays: A unified method of solution, and some extensions. Aequationes Mathematicae, 24:47-65, 1982.

[6] N. J. A. Sloane. The on-line encyclopedia of integer sequences. http://www . research. att.com/ njas/sequences/. 